\title{
EFEITOS DO DÉFICIT HÍDRICO SOBRE A FENOMETRIA E A TECNOLOGIA DE FIBRA DO ALGODOEIRO HERBÁCEO
}

\author{
Bernardo Barbosa da Silva ${ }^{1}$, Cleber Brito de Souza ${ }^{2}$, Tantravahi Venkata Ramana Rao ${ }^{3}$, \\ Pedro Vieira de Azevedo ${ }^{3}$ e José Espínola Sobrinho ${ }^{4}$
}

\begin{abstract}
RESUMO
Este trabalho objetivou analisar os efeitos provocados pelo déficit hídrico em variáveis fenológicas e características tecnológicas da fibra do algodoeiro herbáceo (Gossypium hirsutum r. latifolium Hutch). Realizou-se experimento de campo em Ipanguaçu, RN, no período de agosto a dezembro de 1992. Foram utilizadas duas parcelas experimentais com dois níveis de irrigação, cujos resultados demonstram que a cultivar CNPA $6 \mathrm{H}$ no tratamento não estressado apresentou superioridade em altura de planta, índice de área foliar e fitomassa aérea verde e seca, em relação ao tratamento que sofreu deficiência hídrica. A análise das características tecnológicas da fibra não permitiu evidenciar diferenças devidas aos tratamentos e a cultura apresentou rendimento (algodão em caroço) de 3.960kg.ha-1 e de 2.690 $\mathrm{kg} . \mathrm{ha}^{-1}$ nos tratamentos irrigado e estressado, respectivamente.
\end{abstract}

Palavras-chave: Gossypium hirsutum,índice de área foliar, biomassa, fibra do algodoeiro

\section{WATER DEFICIT EFFECTS ON PHENOLOGY AND FIBER CHARACTERISTICS OF COTTON}

\begin{abstract}
The main objective of this research was to analyze the effects of water deficit on some phenologic and fiber characteristics of a cotton crop (Gossypium hirsutum r. latifolium Hutch). A field experiment was conducted from August to December 1992 in the municipality of Ipanguaçu-RN. Two experimental plots were subjected to two irrigation treatments. The results demonstrate that the height of the plant, fresh and dry biomass and leaf area index were higher in cotton plants under irrigated treatment as compared to treatment of water stress. The yields were $3,960 \mathrm{~kg}^{-\mathrm{ha}^{-1}}$ and 2,690 kg.ha- ${ }^{-1}$ (seed cotton) for irrigated and stressed treatments. The laboratory tests of the fiber showed no significant differences among the variables studied (fiber resistance, maturity, uniformity) for the irrigated and stressed treatments.
\end{abstract}

Key words: Gossypium hirsutum, biomass, leaf area index and cotton fiber

\footnotetext{
${ }^{1}$ Professor, Doutor, Departamento de Ciências Atmosféricas (DCA), Centro de Ciências e Tecnologia da Universidade Federal da Paraíba, Av. Aprígio Veloso 822. CEP 58109-970, Campina Grande, PB. E-mail: bernardo@ dca.ufpb.br

2 Professor, Mestre, Centro de Formação de Tecnólogos da UFPB, Bananeiras, PB

3 Professor, Doutor, DCA/CCT/UFPB, Campina Grande, PB

${ }^{4}$ Professor, Mestre, Depto de Engenharia Agrícola da Escola Superior Agrícola de Mossoró, CEP 59625-900, Mossoró, RN
} 


\section{INTRODUÇÃO}

$\mathrm{O}$ algodoeiro foi, durante muito tempo, a principal cultura de subsistência no semi-árido do Nordeste brasileiro, sendo de capital importância para a economia da região, uma vez que compreendia $25 \%$ da renda agrícola e ocupava aproximadamente $60 \%$ da força de trabalho do campo (Souza, 1986).

Em anos com precipitação normal esta cultura responde significativamente aos insumos e inovações tecnológicas aplicados; contudo, na década de 80 os baixos preços praticados no mercado regional, associados a fenômenos meteorológicos adversos, à praga do bicudo (Antonomus grandis) além de outros fatores, provocaram redução substancial na produtividade e na área plantada.

A microrregião Vale do Açu, no Estado do Rio Grande do Norte, tem sido considerada de grande potencial para a exploração do algodoeiro irrigado. No quinquênio 1988-1993, importantes trabalhos de pesquisa foram desenvolvidos naquele Vale, com a finalidade de incrementar e melhor orientar o plantio do algodão na região (Silva \& Bezerra, 1990; Azevedo et al. 1993).

Esta cultura tem sido bastante estudada em todo o mundo, sendo considerada uma planta muito sensível às variações do conteúdo de água do solo durante e principalmente na floração (Hearn, 1975; Rao et al., 1978). O manejo hídrico inadequado da cultura pode comprometer a sua fibra (Hanson \& Knisel, 1964; Jackson \& Tilt, 1968; Marani \& Amirav, 1971) além de proporcionar alterações significativas na fenologia e na produção (Oliveira, 1979; Karida \& Marinato, 1982; Souza, 1986; Silva \& Bezerra, 1990).

O presente trabalho objetivou avaliar os efeitos do estresse hídrico sobre a fenologia e algumas caraterísticas tecnológicas da fibra do algodoeiro herbáceo, cultivado em condições de clima semi-árido, no Vale do Açu, RN.

\section{MATERIAL E MÉTODOS}

O trabalho foi conduzido na Estação Experimental da Empresa de Pesquisa Agropecuária do Estado do Rio Grande do Norte - EMPARN, localizada no município de Ipanguaçu-RN, com latitude $5^{\circ} 30^{\prime} \mathrm{S}$, longitude $36^{\circ} 55^{\prime} \mathrm{O}$ e altitude $68 \mathrm{~m}$, de agosto a dezembro de 1992. Segundo Medina \& Maia Neto (1989) o clima local é do tipo BSw'h', conforme classificação de Koppen, correspondendo a um clima quente, semi-árido, com estação chuvosa limitada aos meses de fevereiro a maio. As temperaturas são geralmente superiores a $24^{\circ} \mathrm{C}$, alcançando no verão médias de $29^{\circ} \mathrm{C}$. De acordo com SUDENE (1968) os solos da área são pouco desenvolvidos, não hidromórficos e classificados como Aluvial Eutrófico, enquanto a análise textural realizada permitiu classificá-los como franco-argilo-siltoso. A cultivar de algodão herbáceo (Gossypium hirsutum $r$. latifolium Hutch) utilizada foi a CNPA-6H; foram consideradas duas parcelas, medindo $40 \mathrm{~m} \times 30 \mathrm{~m}$, correspondendo cada uma a um tratamento de irrigação. No tratamento estressado (TE) a cultura foi submetida à supressão de água a partir dos 48 dias após a semeadura (DAS ); no tratamento não estressado (TNE) a cultura foi mantida em boas condições de suprimento de água, recebendo duas irrigações semanais com lâmina calculada segundo a evapotranspiração estimada pelo método do Tanque Classe A, utilizando-se coeficiente de cultivo sugerido por Azevedo et al. (1991). Durante o experimento foram relizados o balanço de energia e o monitoramento da temperatura do dossel da cultura, com vistas à quantificação do estresse hídrico, conforme Silva et al. (1995) e, por esta razão e pela dimensão das parcelas, não foi realizada repetição entre os tratamentos. No interior das parcelas de cada tratamento demarcou-se uma área de $10 \mathrm{~m} \mathrm{x}$ 10m para acompanhamento da fenologia e quantificação da produtividade.

A semeadura foi realizada em 28 de agosto de 1992, obtendo-se densidade populacional de aproximadamente 100.000 plantas.ha ${ }^{-1}$, semeadas em fileiras duplas com distância entre linhas simples de $0,35 \mathrm{~m}$ e entre linhas duplas de $0,65 \mathrm{~m}$. Os tratos culturais (adubação, controle de ervas daninhas e pragas) foram efetuados de acordo com recomendações técnicas da EMBRAPA-Algodão, para a região.

O sistema de irrigação utilizado foi o de sulcos fechados nos extremos, com tubos janelados espaçados de $1,0 \mathrm{~m}$ e o controle de água nas parcelas foi feito via hidrômetro; durante o período não foi registrada a ocorrência de chuvas.

Em cada área demarcada foram selecionadas 20 plantas, para o acompanhamento semanal de suas alturas médias; nos dias correspondentes a tais medições eram extraídas três plantas em cada tratamento, para obtenção da área foliar média através de um integrador de área marca LI-3300 da LICOR, fitomassa epígea verde e fitomassa epígea seca em estufa a $70^{\circ} \mathrm{C} . \mathrm{Na}$ colheita foram obtidos 50 capulhos por tratamento para determinação da porcentagem de fibra, comprimento, finura, maturidade e resistência; para tanto, foram usados o HVI (Height Volume Instruments) modelo 900 e o Finurímetro-maturímetro FMT2, ambos pertencentes ao Centro Nacional de Pesquisa de Algodão, da Empresa Brasileira de Pesquisa Agropecuária EMBRAPA, e obedecidos os procedimentos sugeridos por Santana \& Wanderley (1995). As variáveis altura de planta, fitomassas epígeas verde e seca e índice de área foliar, foram submetidas à análise de regressão linear e não-linear, segundo o software Curvefit.

\section{RESULTADOS E DISCUSSÃO}

No tratamento não estressado (TNE) a altura máxima medida foi de $93 \mathrm{~cm}$, embora a média das 20 plantas tenha se situado em torno de $76 \mathrm{~cm}$, a partir do completo crescimento da cultura, verificado aos 86 (DAS). Observou-se uma pequena, porém virtual, diferença entre as alturas médias de plantas no tratamento estressado (TE) e aquelas verificadas no TNE, como pode ser observado na Figura 1. No TE, a altura média situou-se em

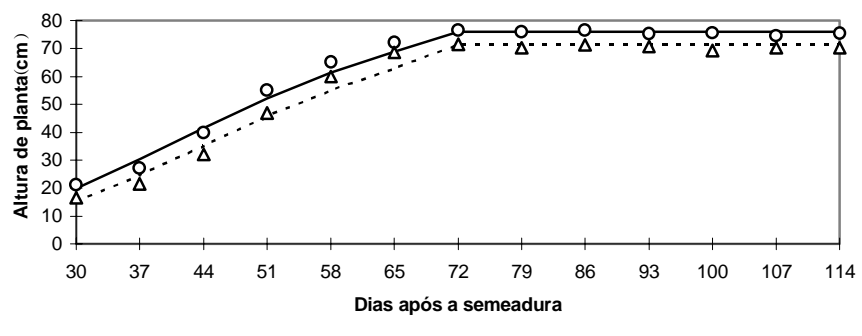

- Medida TNE — Regressão TNE $\Delta \quad$ Medida TE - - - - - Regressão TE

Figura 1. Comportamento estacional da altura média de plantas do algodoeiro herbáceo cultivado sob diferentes tratamentos 
torno dos $70 \mathrm{~cm}$ e ocorreu no $72 \mathrm{DAS}$, enquanto as diferenças observadas entre os tratamentos podem ser atribuídas principalmente ao fator água e à eventual diferença na concentração de sais e adubos no interior das parcelas. Oliveira (1979) também encontrou diferenças significativas na altura do algodoeiro herbáceo entre tratamentos irrigados, em pesquisa conduzida no Estado da Bahia; a estimativa da altura de planta (H) em centímetros, pode ser determinada através das expressões de regressão apresentadas a seguir:

$$
H(T N E)=-22,31+1,44 D A S
$$

em que DAS se refere ao número de dias após a semeadura. A expressão anteriormente citada apresentou coeficiente de determinação igual a 0,9701 ; já no tratamento estressado (TE) obteve-se:

$$
H(T E)=-29,41+1,47 D A S
$$

que resultou num coeficiente de determinação de 0,9702.

Deve-se ressaltar que a utilização das equações retrocolocadas somente se aplica para DAS maiores que 30 e até o dia em que a cultura alcançar a sua altura média máxima.

Os valores da fitomassa epígea verde (FEV) estão apresentados na Figura 2 e as médias, em ambos os tratamentos, foram igualmente submetidas à análise de regressão matemática, resultando nas seguintes equações:

$$
\begin{gathered}
F E V(T N E)=0,4490 \cdot 10^{-9} \cdot 0,9237^{D A S} \cdot D A S^{7,702} \\
F E V(T E)=261,6 \operatorname{EXP}\left[-\frac{(D A S-94,5)^{2}}{1282,0}\right]
\end{gathered}
$$

cujos valores se encontram em g.planta ${ }^{-1}$ e os coeficientes de determinação para os tratamentos TNE e TE foram, respectivamente, iguais a 0,9579 e 0,9546 .

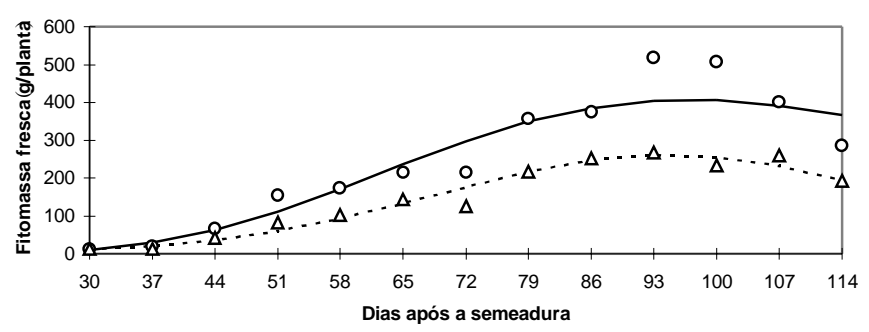

○ Medida TNE $\longrightarrow$ Regressão TNE $\quad \Delta \quad$ Medida TE $\quad \cdots-$ - - - Regressão TE

Figura 2. Comportamento estacional da fitomassa fresca (g/planta) do algodoeiro herbáceo cultivado sob diferentes tratamentos

Observa-se, na Figura 2, que aos 93 DAS a FEV alcançou cerca de 520 g.planta ${ }^{-1}$ no TNE e 270 g.planta ${ }^{-1}$ no outro tratamento e que os valores compreendidos entre os 93 DAS e 100 DAS apresentaram maior dispersão em relação à curva de regressão no TNE, enquanto se obteve, no TE, uma maior concordância entre as observações e a estimativa. As dispersões observadas podem ter resultado da sistemática adotada para a escolha do material. Como se sabe, existe uma distribuição de frequiência associada à fitomassa epígea verde, à fitomassa epígea seca e à área foliar, de plantas de uma mesma cultivar. Por sua vez, a dispersão dos valores da fitomassa epígea verde de uma cultivar em relação à média, resulta de diferentes fatores, destacando-se a desuniformidade da umidade do solo na área plantada, a heterogeneidade do solo e a presença de manchas de solo com elevado teor de sais. Procurou-se minimizar a variabilidade natural da variável ao se coletar plantas com alturas iguais à média das 20 plantas monitoradas no estande de cada tratamento; no entanto, este critério não é sensível a outros fatores, como a variabilidade do número de folhas, fitomassa epígea e área foliar, existentes entre plantas de uma mesma altura; assim sendo, as variações observadas em um mesmo tratamento podem ser atribuídas a tais fatores.

Os dados da fitomassa epígea seca (FES) estão representados na Figura 3. Os dados do TNE apresentaram valor máximo aos 93 DAS que, na ocasião, apresentaram valores da ordem de $120 \mathrm{~g}$. As regressões matemáticas obtidas na caracterização da variação estacional da fitomassa FES nos tratamentos TNE e TE, foram:

$$
\begin{gathered}
F E S(T N E)=0,1291 \cdot 10^{-9} \cdot 0,9346^{D A S} \cdot D A S^{7,428} \\
F E S(T E)=81,02 \cdot \operatorname{EXP}\left[-\frac{(D A S-104,3)^{2}}{1418,0}\right]
\end{gathered}
$$

que proporcionaram coeficientes de determinação iguais, respectivamente, a 0,9651 e 0,9645 .

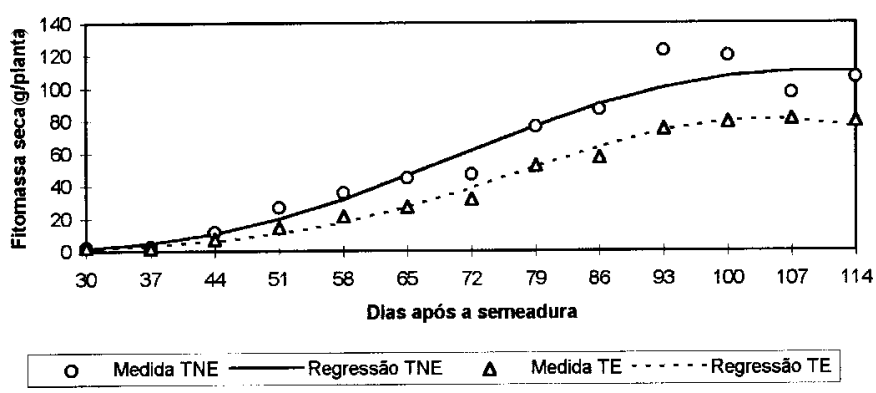

Figura 3. Comportamento estacional da fitomassa seca (g.planta-1) do algodoeiro herbáceo cultivado sob diferentes tratamentos

Para o TE observa-se que os valores apresentam uma sequiência de comportamento mais uniforme, sem maiores dispersões mostrando, assim, o aumento gradativo e as concentrações de assimilados coerentes em todo o ciclo analisado; no TNE, o valor final da FES foi de 106,4 g.planta${ }^{1}$ formando, então, uma geração média semanal de 8,7 g.planta ${ }^{1}$. No TE, como era de se esperar, o acúmulo final de matéria seca alcançou apenas 79,7 g.planta ${ }^{-1}$, com média semanal de 6,4 g.planta ${ }^{-1}$. Observa-se também que tanto a FES não estressada como a FES estressada representaram, até os 65 dias de desenvolvimento da cultura, menos de $20 \%$ da matéria verde acumulada, mas após os 65 dias esse percentual aumentou para $21,6 \%$ para a FES irrigada em relação à FEV não estressada e $24,3 \%$ da FES do tratamento estressado, ambos em relação à matéria verde do tratamento não estressado. 
O índice de área foliar (IAF) do algodoeiro no TNE e no TE, obtido dos 30 aos 114 dias após a semeadura, pode ser observado na Figura 4, na qual são apresentadas as variações estacionais do IAF medido e estimado por regressão matemática. As expressões de regressão matemática que representam cada uma das curvas ajustadas aos tratamentos resultaram nas equações:

$$
\begin{gathered}
\operatorname{IAF}(T N E)=0,132 \cdot 10^{-9} \cdot 0,9227^{D A S} \cdot D A S^{6,923} \\
\operatorname{IAF}(T E)=2,597 . \mathrm{EXP}\left[-\frac{(D A S-84,20)^{2}}{1 \cdot 136,23}\right]
\end{gathered}
$$

com coeficientes de determinação iguais, respectivamente, a 0,9473 e 0,8937 .

O IAF permite avaliar o potencial de rendimento de uma cultura, com base no aproveitamento da radiação fotossinteticamente ativa. O IAF e a área foliar são numericamente diferentes, porém ambos refletem a eficiência no aproveitamento da luz e/ou dos nutrientes do solo.

Tanto nas condições do TNE como nas do TE, a cultura atingiu o maior IAF aos 93 dias, ou 14 semanas (Figura 4), enquanto os valores máximos foram de $3,89 \mathrm{~m}^{2} . \mathrm{m}^{-2}$ e de $3,23 \mathrm{~m}^{2} . \mathrm{m}^{-2}$, para TNE e TE, respectivamente. Ao se considerar as curvas de ajustamento, percebe-se que há uma virtual diferença entre os tratamentos e após o máximo associado ao TE, acentuam-se as diferenças do IAF entre os tratamentos, o que pode ser atribuído ao estresse hídrico.

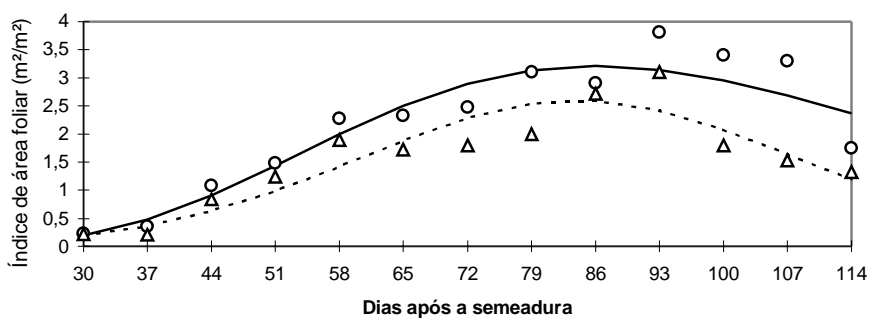

○ Medida TNE — Regressão TNE $\quad \Delta \quad$ Medida TE - - - - - Regressão TE

Figura 4. Comportamento estacional do índice de área foliar $\left(\mathrm{m}^{2} \cdot \mathrm{m}^{-2}\right)$ do algodoeiro herbáceo cultivado sob diferentes tratamentos

Os dados relativos à massa do caroço mais pluma no TNE apresentaram diferença de 35,3g, em comparação ao TE (Tabela 1); da mesma forma, observou-se no TNE tendência de superioridade em peso de pluma, peso de 100 sementes, porcentagem de fibra e peso médio de capulho, quando comparado ao TE. No parâmetro porcentagem de semente, o TE apresentou tendência ligeiramente superior ao TNE. De certa forma, a cultivar CNPA $6 \mathrm{H}$ apresentou peso médio de capulho no intervalo de $5,5 \mathrm{~g}$ a $6,5 \mathrm{~g}$, dados esses também confirmados por Barreto (1993) ao trabalhar com esta cultivar em condições

Tabela 1. Componentes de produção do algodoeiro herbáceo nos tratamentos não estressado (TNE) e estressado (TE) em Ipanguaçu, RN, 1992

\begin{tabular}{ccccccc}
\hline Tratamento & & Peso (g) & \multicolumn{2}{c}{ Peso (\%) } & Peso/capulho (g) \\
\cline { 1 - 6 } TNE & caroço & pluma & 100 sem. & fibra & semente & \\
\cline { 2 - 6 } TE & 326,8 & 136,8 & 10,8 & 41,9 & 58,1 & 6,5 \\
\hline
\end{tabular}

irrigadas, no município de Ipanguaçu.

No que diz respeito ao rendimento da cultura, o TNE apresentou produtividade de $3.960 \mathrm{~kg} \cdot \mathrm{ha}^{-1}$ (algodão em caroço), enquanto no TE o rendimento foi de $2.690 \mathrm{~kg} . \mathrm{ha}^{-1}$. Como se esperava, o TNE, como um todo, apresentou tendência de superioridade ao TE, concluindo-se que as ótimas condições de disponibilidade de água oferecida à cultura durante todas as fases de crescimento e desenvolvimento, são primordiais para a obtenção de melhores rendimentos.

$\mathrm{Na}$ Tabela 2 estão representadas algumas características físicas das fibras do algodoeiro. Com relação ao comprimento de fibra, a Span Length (SL) 2,5\%, obtiveram-se 29,2 mm no TNE e 29,9 mm no TE; numa classificação comercial, as fibras com comprimento entre $29,2 \mathrm{~mm}$ e $29,9 \mathrm{~mm}$ são tidas como médias (Santana \& Wanderley, 1995) de forma que não houve diferença no comprimento das fibras devido aos tratamentos aplicados. Uma característica marcante da cultivar CNPA 6H é a sua uniformidade de fibra (Freire et al., 1997). Como se observa na Tabela 2, os valores obtidos no TNE foram de 52,7\% e no TE de 56,2\%, correspondendo à categoria "muito uniforme"; em tal sistema de classificação valores acima de $46 \%$ correspondem a fibras "muito uniformes".

Tabela 2. Resultados das análises tecnológicas da fibra do algodoeiro herbáceo nos tratamentos TNE e TE em Ipanguaçu, RN, 1992

\begin{tabular}{ccccccccc}
\hline Tratamento & \multicolumn{3}{c}{ Comprimento } & \multicolumn{2}{c}{ Finura } & \multicolumn{2}{c}{ Maturidade } & Resistencia \\
\cline { 2 - 9 } & SL50\% & $\begin{array}{c}\text { SL2,5(\%) } \\
(\mathrm{mm})\end{array}$ & $\%$ UNIF & $\begin{array}{c}\text { MIC } \\
(\mu \mathrm{g} / \mathrm{in})\end{array}$ & $\begin{array}{c}\text { Fin } \\
(\mathrm{mtex})\end{array}$ & $\begin{array}{c}\text { Mat } \\
\text { Lord }\end{array}$ & $\begin{array}{c}\text { Pm } \\
\text { ASTM } \%\end{array}$ & $\begin{array}{c}\text { Press } \\
(\mathrm{lb} / \mathrm{mg})\end{array}$ \\
\hline TNE & 15,4 & 29,2 & 52,7 & 4,1 & 194 & 0,77 & 68,5 & 8,0 \\
TE & 16,8 & 29,9 & 56,2 & 4,3 & 193 & 0,84 & 75,3 & 7,6 \\
\hline
\end{tabular}

Utilizou-se o finurímetro-maturímetro FMT2 e os resultados obtidos permitem classificar as fibras obtidas em ambos os tratamentos como de "finura média", uma vez que as fibras no TNE e TE obtiveram valores iguais, respectivamente, a 4,1 mg.pol ${ }^{-1}$ e 4,3 mg.pol ${ }^{-1}$, valores esses próximos àqueles de alta demanda da indústria nacional, conforme Freire et al. (1997).

Quanto à maturidade, os valores encontrados estiveram entre $68 \%$ e $76 \%$, o que os classifica como de "maturidade ASTN média" (Santana \& Wanderley, 1995).

Também de grande importância para a indústria têxtil, são as análises de resistência das fibras. Medido em Índice Presley (lb.mg-1) este parâmetro apresentou 8,0 lb. $\mathrm{mg}^{-1}$ no TNE e 7,6 lb. $\mathrm{mg}^{-1}$ no TE, valores esses compreendidos numa faixa de classificação entre 7,5 a 8,15 lb. $\mathrm{mg}^{-1}$, tidas como de "resistência média”, ainda segundo Santana \& Wanderley (1995).

Conclui-se que os déficits de água ocorridos não alteraram maturidade, comprimento, finura nem resistência das fibras, porém Marani \& Amirav (1971) concluíram que o índice e o comprimento das fibras do algodoeiro foram afetados pelo estresse hídrico verificado na segunda metade da floração. Para Marani (1973) o estresse hídrico também não afetou a resistência das fibras. Deve-se observar, no entanto, que os efeitos produzidos pelo estresse hídrico nas variáveis citadas estão a depender de sua duração e intensidade.

\section{CONCLUSÃO}

O algodoeiro herbáceo, cv. CNPA $6 \mathrm{H}$, cultivado no tratamento não estressado, apresentou superioridade em altura de planta, área foliar, fitomassa e rendimento, em relação aos 
valores obtidos no tratamento estressado. Os componentes tecnológicos da fibra da cultura considerados na pesquisa receberam a mesma classificação de finura, maturidade, resistência e comprimento.

\section{AGRADECIMENTOS}

Os autores agradecem a colaboração do Centro Nacional de Pesquisa de Algodão - CNPA, da EMBRAPA - Algodão, da Escola Superior Agrícola de Mossoró - ESAM e da Empresa de Pesquisa Agropecuária do Rio Grande do Norte - EMPARN, pela utilização de suas áreas experimentais, equipamentos e laboratórios.

\section{REFERÊNCIAS BIBLIOGRÁFICAS}

AZEVEDO, P.V; RAMANA RAO, T.V.; AMORIM, S.A.; BEZERRA, J.R.C.; ESPÍNOLA SOBRINHO, J. Estimativa do consumo hídrico de um cultivo de algodão herbáceo. IN: CONGRESSO BRASILEIRODE AGROMETEOROLOGIA, 7. Viçosa. Resumos ..., p.229-231, 1991.

AZEVEDO, P.V.; SILVA, B.B.; RAMANA RAO, T.V.; FROTA, R.N.B.; ESPÍNOLA SOBRINHO, J. Modelos de estimativa da área foliar do algodoeiro herbáceo. IN: CONGRESSO BRASILEIRO DE AGROMETEOROLOGIA, 8., 1993. Porto Alegre. Resumos... Santa Maria, Rio Grande do Sul: Sociedade Brasileira de Agrometeorologia: p.197, 1993.

BARRETO, A.N. Relatório preliminar sobre o desempenho do algodoeiro herbáceo, cultivar CNPA $6 \mathrm{H}$, em condições irrigadas no município de Ipanguaçu, RN. Campina Grande-PB, EMBRAPA-Algodão, 52 p., 1993.

FREIRE, E.C.; SOARES, J.J.; FARIAS, F.J.C.; ARANTES, E.M.; ANDRADE, F.P. de; PARO, H.; LACA-BUENDIA, J.P. Cultivo do algodoeiro no Estado do Mato Grosso. Campina Grande: EMBRAPA-CNPA, 1997, 65p (EMBRAPA-CNPA, Circular Técnico, 23).

HANSON, E.G.; KNISEL, W.G. Influence of irrigation practices on cotton production and fiber properties. New Mexico: Agricultural Experimental Station, 1964. 34p (Bull, 43).

HEARN, A.B. Response of cotton to water and nitrogen in tropical environment. I. Frequency of watering and method of application of nitrogen, Journal of Agricultural Science, v.84, p.407-417, 1975.
JACKSON, L.E.B.; TILT, P.A. Effects of irrigation intensity and nitrogen level on the performance of eight varieties of upland cotton (gossypium hirsutum L.). Agronomy Journal, v.60, p.13-17, 1968

KARIDA, J.; MARINATO, R. Irrigação do algodoeiro. Informe Agropecuário. Belo Horizonte, v.8, n.92, p.75-81, 1982.

MARANI, A. Effects of soil moisture stress on two varieties of upland cotton in Israel. IV. Effects of periods of stress ocurrence, correlations and regressions. Experimental Agriculture, v.9, n.2, p.121-129, 1973.

MARANI, A.; AMIRAV, A. Effects of soil moisture stress on two varieties of upland cotton in Israel. I - The coastal plain region. Experimental Agriculture, v.7, p.213-24, 1971.

MEDINA, B.F.; MAIA NETO, J.M. Estudo da precipitação pluviométrica no Estado do Rio Grande do Norte. Coleção Mossoroense, 66p, 1989.

OLIVEIRA, F.A. Efeitos dos diferentes níveis de umidade do solo no ciclo e produtividade no algodão herbáceo (Gossypium hirsutumb L.). Salvador: EPABA, 1979. 26p. (EPABA, Comunicado Técnico,7.)

RAO, G.R.; SHIDE, J.S.; KADAM, D.M.; VARADE, S.B. Phenoclimatological study of rainfed cotton crop. International Journal of Ecology and Environment Science, v.4, p.7-13, 1978.

SANTANA, J.C.F.; WANDERLEY, M.J.R. Interpretação de resultados de análise de fibra, efetuadas pelo instrumento de alto volume (HVI) e pelo finurímetro-maturímetro (FMT2). Campina Grande: EMBRAPA-CNPA, 1995. 9p. (EMBRAPA-CNPA. Comunicado Técnico, 41).

SILVA, M.J. da; BEZERRA, J.R.C. Manejo de irrigação na cultura do algodoeiro no Vale do Açu-RN. REUNIÃO NACIONAL DO ALGODÃO, 6., 1990, Campina Grande, PB. 1990. Resumo dos Trabalhos. Campina Grande: EMBRAPA-CNPA, 1990.

SILVA, B.B. da; RAMANA RAO, T.V.; AZEVEDO, P.V.; ESPÍNOLA SOBRINHO, J. Quantificação de estresse hídrico em algodoeiro herbáceo com termometria infravermelha. Revista Brasileira de Agrometeorologia, v.3, p.45-51, 1995.

SOUZA, F.A. Efeito do estresse hídrico e da época de plantio na produtividade de três cultivares de algodão herbáceo (Gossypium hirsutum L.) sob regime de irrigação no vale do Curu. Fortaleza, UFCE, 1986, 90p. (Dissertação de Mestrado).

SUDENE. Mapa Exploratório do Estado do Rio Grande do Norte-RN,1968. 87p. 